\title{
LAGRANGE'S THEOREM FOR HOPF MONOIDS IN SPECIES
}

\author{
MARCELO AGUIAR AND AARON LAUVE
}

\begin{abstract}
Following Radford's proof of Lagrange's theorem for pointed Hopf algebras, we prove Lagrange's theorem for Hopf monoids in the category of connected species. As a corollary, we obtain necessary conditions for a given subspecies $\mathbf{k}$ of a Hopf monoid $\mathbf{h}$ to be a Hopf submonoid: the quotient of any one of the generating series of $\mathbf{h}$ by the corresponding generating series of $\mathbf{k}$ must have nonnegative coefficients. Other corollaries include a necessary condition for a sequence of nonnegative integers to be the dimension sequence of a Hopf monoid in the form of certain polynomial inequalities, and of a set-theoretic Hopf monoid in the form of certain linear inequalities. The latter express that the binomial transform of the sequence must be nonnegative.
\end{abstract}

\section{INTRODUCTION}

Lagrange's theorem states that for any subgroup $K$ of a group $H, H \cong K \times Q$ as (left) $K$-sets, where $Q=H / K$. In particular, if $H$ is finite, then $|K|$ divides $|H|$. Passing to group algebras over a field $\mathbb{k}$, we have that $\mathbb{k} H \cong \mathbb{k} K \otimes \mathbb{k} Q$ as (left) $\mathbb{k} K$-modules, or that $\mathbb{k} H$ is free as a $\mathbb{k} K$-module. Kaplansky [10] conjectured that the same statement holds for Hopf algebras - group algebras being principal examples. It turns out that the result does not hold in general, as shown by Oberst and Schneider [21, Proposition 10] and [18, Example 3.5.2]. On the other hand, the result does hold for certain large classes of Hopf algebras, including the finite dimensional ones by a theorem of Nichols and Zoeller [20], and the pointed ones by a theorem of Radford [22]. Further (and finer) results of this nature were developed by Schneider [27, 28]. Additional work on the conjecture includes that of Masuoka [16] and Takeuchi [32]; more information can be found in Sommerhäuser's survey [30].

The main result of this paper (Theorem 7) is a version of Lagrange's theorem for Hopf monoids in the category of connected species: if $\mathbf{h}$ is a connected Hopf monoid and $\mathbf{k}$ is a Hopf submonoid, there exists a species $\mathbf{q}$ such that $\mathbf{h}=\mathbf{k} \cdot \mathbf{q}$. An immediate application is a test for Hopf submonoids (Corollary 13): if any one of the generating series for a species $\mathbf{k}$ does not divide in $\mathbb{Q} \geq 0 \llbracket x \rrbracket$ the corresponding generating series for the Hopf monoid $\mathbf{h}$ (in the sense that the quotient has at least one negative coefficient), then $\mathbf{k}$ is not a Hopf submonoid of $\mathbf{h}$. A similar test also holds for connected graded

Date: 18 August 2011.

2010 Mathematics Subject Classification. 05A15; 05A20; 05E99; 16T05; 16T30; 18D10; 18 D35.

Key words and phrases. Hopf monoids, species, graded Hopf algebras, Lagrange's theorem, generating series, Poincaré-Birkhoff-Witt theorem, Hopf kernel, Lie kernel, primitive element, partition, composition, linear order, cyclic order, derangement.

Aguiar supported in part by NSF grant DMS-1001935.

Lauve supported in part by NSA grant H98230-11-1-0185. 
Hopf algebras (Corollary 4). The proof of Theorem 7 for Hopf monoids in species parallels Radford's proof for Hopf algebras. (Hopf algebras are Hopf monoids in the category of vector spaces).

The paper is organized as follows. In Section 1, we recall Lagrange's theorem for Hopf algebras, focusing on the case of connected graded Hopf algebras. In Section 2, we recall the basics of Hopf monoids in species and prove Lagrange's theorem in this setting. Examples and applications are given in Section 3. Among these, we derive certain polynomial inequalities that a sequence of nonnegative integers must satisfy in order to be the dimension sequence of a connected Hopf monoid in species. In the case of a set-theoretic Hopf monoid structure, we obtain additional necessary conditions in the form of linear inequalities which express that the binomial transform of the enumerating sequence must be nonnegative. In Section 4 we provide information on the growth and support of the dimension sequence of a connected Hopf monoid. The latter must be an additive submonoid of the natural numbers. We conclude in Section 5 with information on the species q entering in Lagrange's theorem. In the dual setting, $\mathbf{q}$ is the Hopf kernel of a morphism, and for cocommutative Hopf monoids it can be described in terms of Lie kernels and primitive elements via the Poincaré-Birkhoff-Witt theorem.

All vector spaces are over a fixed field $\mathbb{k}$ of characteristic 0, except in Section 4, where the characteristic is arbitrary.

\section{LAGRANGE'S THEOREM FOR HOPF ALGEBRAS}

We begin by recalling a couple of versions of this theorem.

Theorem 1. Let $H$ be a finite dimensional Hopf algebra over a field $\mathbb{k}$. If $K \subseteq H$ is any Hopf subalgebra, then $H$ is a free left (and right) $K$-module.

This is the Nichols-Zoeller theorem [20]; see also [18, Theorem 3.1.5]. We will not make direct use of this result, but instead of the related results discussed below.

A Hopf algebra $H$ is pointed if all its simple subcoalgebras are 1-dimensional. Equivalently, the group-like elements of $H$ linearly span the coradical of $H$.

Given a subspace $K$ of $H$, set

$$
K_{+}:=K \cap \operatorname{ker}(\epsilon)
$$

where $\epsilon: H \rightarrow \mathbb{k}$ is the counit of $H$. Let $K_{+} H$ denote the right $H$-ideal generated by $K_{+}$.

Theorem 2. Let $H$ be a pointed Hopf algebra. If $K \subseteq H$ is any Hopf subalgebra, then $H$ is a free left (and right) $K$-module. Moreover,

$$
H \cong K \otimes\left(H / K_{+} H\right)
$$

as left $K$-modules.

The first statement is due to Radford [22, Section 4] and the second (stronger) statement to Schneider [27, Remark 4.14], [28, Corollary 4.3]. Various generalizations can be found in these references as well as in Masuoka [16] and Takeuchi [32]; see also Sommerhaüser [30]. We are interested in the particular variant given in Theorem 3 below. 
A Hopf algebra $H$ is graded if there is given a decomposition

$$
H=\bigoplus_{n \geq 0} H_{n}
$$

into linear subspaces that is preserved by all operations. It is connected graded if in addition $H_{0}$ is linearly spanned by the unit element.

Theorem 3. Let $H$ be a connected graded Hopf algebra. If $K \subseteq H$ is a graded Hopf subalgebra, then $H$ is a free left (and right) $K$-module. Moreover,

$$
H \cong K \otimes\left(H / K_{+} H\right)
$$

as left $K$-modules and as graded vector spaces.

Proof. Since $H$ is connected graded, its coradical is $H_{0}=\mathbb{k}$, so $H$ is pointed and Theorem 2 applies. Radford's proof shows that there exists a graded vector space $Q$ such that

$$
H \cong K \otimes Q
$$

as left $K$-modules and as graded vector spaces. (The argument we give in the parallel setting of Theorem 7 makes this clear.) Note that $K_{+}=\bigoplus_{n \geq 1} K_{n}$, hence $K_{+} H$ and $H / K_{+} H$ inherit the grading of $H$. To complete the proof, it suffices to show that $Q \cong H / K_{+} H$ as graded vector spaces.

Let $\varphi: K \otimes Q \rightarrow H$ be an isomorphism of left $K$-modules and of graded vector spaces. We claim that

$$
\varphi\left(K_{+} \otimes Q\right)=K_{+} H
$$

In fact, since $\varphi$ is a morphism of left $K$-modules,

$$
\varphi\left(K_{+} \otimes Q\right)=K_{+} \varphi(1 \otimes Q) \subseteq K_{+} H .
$$

Conversely, if $k \in K_{+}$and $h \in H$, writing $h=\sum_{i} \varphi\left(k_{i} \otimes q_{i}\right)$ with $k_{i} \in K$ and $q_{i} \in Q$, we obtain

$$
k h=\sum_{i} \varphi\left(k k_{i} \otimes q_{i}\right) \in \varphi\left(K_{+} \otimes Q\right),
$$

since $K_{+}$is an ideal of $K$.

Now, since $K=K_{0} \oplus K_{+}$, we have

$$
K \otimes Q=\left(K_{0} \otimes Q\right) \oplus\left(K_{+} \otimes Q\right)
$$

and therefore

$$
H / K_{+} H=\varphi(K \otimes Q) / \varphi\left(K_{+} \otimes Q\right) \cong \varphi\left(K_{0} \otimes Q\right) \cong Q
$$

as graded vector spaces.

Given a graded Hopf algebra $H$, let $\mathcal{O}_{H}(x) \in \mathbb{N} \llbracket x \rrbracket$ denote its Poincaré series - the ordinary generating function for the sequence of dimensions of its graded components,

$$
\mathcal{O}_{H}(x):=\sum_{n \geq 0} \operatorname{dim} H_{n} x^{n} .
$$

Suppose $H$ is connected graded and $K$ is a graded Hopf subalgebra. In this case, their Poincaré series are of the form

$$
1+a_{1} x+a_{2} x^{2}+\cdots
$$


with $a_{i} \in \mathbb{N}$ and the quotient $\mathcal{O}_{H}(x) / \mathcal{O}_{K}(x)$ is a well-defined power series in $\mathbb{Z} \llbracket x \rrbracket$.

Corollary 4. Let $H$ be a connected graded Hopf algebra. If $K \subseteq H$ is any graded Hopf subalgebra, then the quotient $\mathcal{O}_{H}(x) / \mathcal{O}_{K}(x)$ of Poincaré series is nonnegative, i.e., belongs to $\mathbb{N} \llbracket x \rrbracket$.

Proof. By Theorem 3, $H \cong K \otimes Q$ as graded vector spaces, where $Q=H / K_{+} H$. Hence $\mathcal{O}_{H}(x)=\mathcal{O}_{K}(x) \mathcal{O}_{Q}(x)$ and the result follows.

Example 5. Consider the Hopf algebra QSym of quasisymmetric functions in countably many variables, and the Hopf subalgebra Sym of symmetric functions. They are connected graded, so by Theorem 3, QSym is a free module over Sym. Garsia and Wallach prove this same fact for the algebras $Q S y m_{n}$ and $S y m_{n}$ of (quasi) symmetric functions in $n$ variables (where $n$ is a finite number) [7]. While $Q S y m_{n}$ and $S_{y m}$ are quotient algebras of QSym and Sym, they are not quotient coalgebras. Since a Hopf algebra structure is needed in order to apply Theorem 3, we cannot derive the result of Garsia and Wallach in this manner. The papers [7] and [12] provide information on the space $Q_{n}$ entering in the decomposition $Q S y m_{n} \cong S_{y m_{n}} \otimes Q_{n}$.

\section{LAGRANGE'S THEOREM FOR HOPF MONOIDS IN SPECIES}

We first review the notion of Hopf monoid in the category of species, following [2], and then prove Lagrange's theorem in this setting. We restrict attention to the case of connected Hopf monoids.

2.1. Hopf monoids in species. The notion of species was introduced by Joyal [9]. It formalizes the notion of combinatorial structure and provides a framework for studying the generating functions which enumerate these structures. The book [4] by Bergeron, Labelle and Leroux expounds the theory of set species.

Joyal's work indicates that species may also be regarded as algebraic objects; this is the point of view adopted in [2] and in this work. To this end, it is convenient to work with vector species.

A (vector) species is a functor $\mathbf{q}$ from finite sets and bijections to vector spaces and linear maps. Specifically, it is a family of vector spaces $\mathbf{q}[I]$, one for each finite set $I$, together with linear maps $\mathbf{q}[\sigma]: \mathbf{q}[I] \rightarrow \mathbf{q}[J]$, one for each bijection $\sigma: I \rightarrow J$, satisfying

$$
\mathbf{q}\left[\mathrm{id}_{I}\right]=\mathrm{id}_{\mathbf{q}[I]} \quad \text { and } \mathbf{q}[\sigma \circ \tau]=\mathbf{q}[\sigma] \circ \mathbf{q}[\tau]
$$

whenever $\sigma$ and $\tau$ are composable bijections.

The notation $\mathbf{q}[a, b, c, \ldots]$ is shorthand for $\mathbf{q}[\{a, b, c, \ldots\}]$ and $\mathbf{q}[n]$ is shorthand for $\mathbf{q}[1,2, \ldots, n]$. The space $\mathbf{q}[n]$ is an $S_{n}$-module via $\sigma \cdot v=\mathbf{q}[\sigma](v)$ for $v \in \mathbf{q}[n]$ and $\sigma \in S_{n}$.

A species $\mathbf{q}$ is finite dimensional if each vector space $\mathbf{q}[I]$ is finite dimensional. In this paper, all species are finite dimensional.

A morphism of species is a natural transformation of functors. Let Sp denote the category of (finite dimensional) species.

We give two elementary examples that will be useful later.

Example 6. Let $\mathbf{E}$ be the exponential species, defined by $\mathbf{E}[I]=\mathbb{k}\left\{*_{I}\right\}$ for all $I$. The symbol $*_{I}$ denotes an element canonically associated to the set $I$ (for definiteness, we 
may take $\left.*_{I}=I\right)$. Thus, $\mathbf{E}[I]$ is a 1-dimensional space with a distinguished basis element. A richer example is provided by the species $\mathbf{L}$ of linear orders, defined by $\mathbf{L}[I]=\mathbb{k}\{$ linear orders on $I\}$ for all $I$ (a space of dimension $n$ ! when $|I|=n$ ).

We use $\cdot$ to denote the Cauchy product of two species. Specifically,

$$
(\mathbf{p} \cdot \mathbf{q})[I]:=\bigoplus_{S \sqcup T=I} \mathbf{p}[S] \otimes \mathbf{q}[T] \quad \text { for all finite sets } I .
$$

The notation $S \sqcup T=I$ indicates that $S \cup T=I$ and $S \cap T=\emptyset$. The sum runs over all such ordered decompositions of $I$, or equivalently over all subsets $S$ of $I$ : there is one term for $S \sqcup T$ and another for $T \sqcup S$. The Cauchy product turns Sp into a symmetric monoidal category. The braiding simply switches the tensor factors. The unit object is the species 1 defined by

$$
\mathbf{1}[I]:= \begin{cases}\mathbb{k} & \text { if } I \text { is empty } \\ 0 & \text { otherwise. }\end{cases}
$$

A monoid in the category $(\mathrm{Sp}, \cdot)$ is a species $\mathbf{m}$ together with a morphism of species $\mu: \mathbf{m} \cdot \mathbf{m} \rightarrow \mathbf{m}$, i.e., a family of maps

$$
\mu_{S, T}: \mathbf{m}[S] \otimes \mathbf{m}[T] \rightarrow \mathbf{m}[I]
$$

one for each ordered decomposition $I=S \sqcup T$, satisfying appropriate associativity and unital conditions, and naturality with respect to bijections. Briefly, to each $\mathbf{m}$-structure on $S$ and $\mathbf{m}$-structure on $T$, there is assigned an $\mathbf{m}$-structure on $S \sqcup T$. The analogous object in the category of graded vector spaces is a graded algebra.

For the species $\mathbf{E}$, a monoid structure is defined by sending the basis element $*_{S} \otimes *_{T}$ to the basis element $*_{I}$. For $\mathbf{L}$, a monoid structure is provided by concatenation of linear orders: $\mu_{S, T}\left(\ell_{1} \otimes \ell_{2}\right)=\left(\ell_{1}, \ell_{2}\right)$.

A comonoid in the category $(\mathrm{Sp}, \cdot)$ is a species $\mathbf{c}$ together with a morphism of species $\Delta: \mathbf{c} \rightarrow \mathbf{c} \cdot \mathbf{c}$, i.e., a family of maps

$$
\Delta_{S, T}: \mathbf{c}[I] \rightarrow \mathbf{c}[S] \otimes \mathbf{c}[T]
$$

one for each ordered decomposition $I=S \sqcup T$, which are natural, coassociative and counital.

For the species $\mathbf{E}$, a comonoid structure is defined by sending the basis vector $*_{I}$ to the basis vector $*_{S} \otimes *_{T}$. For $\mathbf{L}$, a comonoid structure is provided by restricting a total order $\ell$ on $I$ to total orders on $S$ and $T: \Delta_{S, T}(\ell)=\left.\left.\ell\right|_{S} \otimes \ell\right|_{T}$.

We assume that our species $\mathbf{q}$ are connected, i.e., $\mathbf{q}[\emptyset]=\mathbb{k}$. In this case, the (co)unital conditions for a (co)monoid force the maps $\mu_{S, T}\left(\Delta_{S, T}\right)$ to be the canonical identifications if either $S$ or $T$ is empty. Thus, in defining a connected (co)monoid structure one only needs to specify the maps $\mu_{S, T}\left(\Delta_{S, T}\right)$ when both $S$ and $T$ are nonempty.

A Hopf monoid in the category $(\mathrm{Sp}, \cdot)$ is a monoid and comonoid whose two structures are compatible in an appropriate sense, and which carries an antipode. In this paper we only consider connected Hopf monoids. For such Hopf monoids, the existence of the antipode is guaranteed. The species $\mathbf{E}$ and $\mathbf{L}$, with the structures outlined above, are two important examples of connected Hopf monoids. 
For further details on Hopf monoids in species, see Chapter 8 of [2]. The theory of Hopf monoids in species is developed in Part II of this reference; several examples are discussed in Chapters 12 and 13.

2.2. Lagrange's theorem for connected Hopf monoids. Given a connected Hopf monoid $\mathbf{k}$ in species, we let $\mathbf{k}_{+}$denote the species defined by

$$
\mathbf{k}_{+}[I]= \begin{cases}\mathbf{k}[I] & \text { if } I \neq \emptyset, \\ 0 & \text { if } I=\emptyset .\end{cases}
$$

If $\mathbf{k}$ is a submonoid of a monoid $\mathbf{h}$, then $\mathbf{k}_{+} \mathbf{h}$ denotes the right ideal of $\mathbf{h}$ generated by $\mathbf{k}_{+}$. In other words,

$$
\left(\mathbf{k}_{+} \mathbf{h}\right)[I]=\sum_{\substack{S \sqcup T=I \\ S \neq \emptyset}} \mu_{S, T}(\mathbf{k}[S] \otimes \mathbf{h}[T]) .
$$

Theorem 7. Let $\mathbf{h}$ be a connected Hopf monoid in the category of species. If $\mathbf{k}$ is a Hopf submonoid of $\mathbf{h}$, then $\mathbf{h}$ is a free left $\mathbf{k}$-module. Moreover,

$$
\mathbf{h} \cong \mathbf{k} \cdot\left(\mathbf{h} / \mathbf{k}_{+} \mathbf{h}\right)
$$

as left $\mathbf{k}$-modules (and as species).

The proof is given after a series of preparatory results. Our argument parallels Radford's proof of the first statement in Theorem 2 [22, Section 4]. The main ingredient is a result of Larson and Sweedler [11] known as the fundamental theorem of Hopf modules [18, Theorem 1.9.4]. It states that if $(M, \rho)$ is a left Hopf module over $K$, then $M$ is free as a left $K$-module and in fact is isomorphic to the Hopf module $K \otimes Q$, where $Q$ is the space of coinvariants for the coaction $\rho: M \rightarrow K \otimes M$. Takeuchi extends this result to the context of Hopf monoids in a braided monoidal category with equalizers [33, Theorem 3.4]; a similar result (in a more restrictive setting) is given by Lyubashenko [14, Theorem 1.1]. As a special case of Takeuchi's result, we have the following.

Proposition 8. Let $\mathbf{m}$ be a left Hopf module over a connected Hopf monoid $\mathbf{k}$ in species. There is an isomorphism $\mathbf{m} \cong \mathbf{k} \cdot \mathbf{q}$ of left Hopf modules, where

$$
\mathbf{q}[I]:=\left\{m \in \mathbf{m}[I] \mid \rho_{S, T}(m)=0 \text { for } S \sqcup T=I, T \neq I\right\} .
$$

In particular, $\mathbf{m}$ is free as a left $\mathbf{k}$-module.

Here $\rho: \mathbf{m} \rightarrow \mathbf{k} \cdot \mathbf{m}$ denotes the comodule structure, which consists of maps

$$
\rho_{S, T}: \mathbf{m}[I] \rightarrow \mathbf{k}[S] \otimes \mathbf{m}[T],
$$

one for each ordered decomposition $I=S \sqcup T$.

Given a comonoid $\mathbf{h}$ and two subspecies $\mathbf{u}, \mathbf{v} \subseteq \mathbf{h}$, the wedge of $\mathbf{u}$ and $\mathbf{v}$ is the subspecies $\mathbf{u} \wedge \mathbf{v}$ of $\mathbf{h}$ defined by

$$
\mathbf{u} \wedge \mathbf{v}:=\Delta^{-1}(\mathbf{u} \cdot \mathbf{h}+\mathbf{h} \cdot \mathbf{v})
$$

The remaining ingredients needed for the proof are supplied by the following lemmas.

Lemma 9. Let $\mathbf{h}$ be a comonoid in species. If $\mathbf{u}$ and $\mathbf{v}$ are subcomonoids of $\mathbf{h}$, then: 
(i) $\mathbf{u} \wedge \mathbf{v}$ is a subcomonoid of $\mathbf{h}$ and $\mathbf{u}+\mathbf{v} \subseteq \mathbf{u} \wedge \mathbf{v}$;

(ii) $\mathbf{u} \wedge \mathbf{v}=\Delta^{-1}(\mathbf{u} \cdot(\mathbf{u} \wedge \mathbf{v})+(\mathbf{u} \wedge \mathbf{v}) \cdot \mathbf{v})$.

Proof. (i) The proofs of the analogous statements for coalgebras given in [1, Section 3.3] extend to this setting.

(ii) From the definition, $\Delta^{-1}(\mathbf{u} \cdot(\mathbf{u} \wedge \mathbf{v})+(\mathbf{u} \wedge \mathbf{v}) \cdot \mathbf{v}) \subseteq \mathbf{u} \wedge \mathbf{v}$. Now, since $\mathbf{u} \wedge \mathbf{v}$ is a subcomonoid,

$$
\Delta(\mathbf{u} \wedge \mathbf{v}) \subseteq((\mathbf{u} \wedge \mathbf{v}) \cdot(\mathbf{u} \wedge \mathbf{v})) \cap(\mathbf{u} \cdot \mathbf{h}+\mathbf{h} \cdot \mathbf{v})=\mathbf{u} \cdot(\mathbf{u} \wedge \mathbf{v})+(\mathbf{u} \wedge \mathbf{v}) \cdot \mathbf{v}
$$

since $\mathbf{u}, \mathbf{v} \subseteq \mathbf{u} \wedge \mathbf{v}$. This proves the converse inclusion.

Lemma 10. Let $\mathbf{h}$ be a Hopf monoid in species and $\mathbf{k}$ be a submonoid. Let $\mathbf{u}, \mathbf{v} \subseteq \mathbf{h}$ be subspecies which are left $\mathbf{k}$-submodules of $\mathbf{h}$. Then $\mathbf{u} \wedge \mathbf{v}$ is a left $\mathbf{k}$-submodule of $\mathbf{h}$.

Proof. Since $\mathbf{h}$ is a Hopf monoid, the coproduct $\Delta: \mathbf{h} \rightarrow \mathbf{h} \cdot \mathbf{h}$ is a morphism of left $\mathbf{h}$-modules, where $\mathbf{h}$ acts on $\mathbf{h} \cdot \mathbf{h}$ via $\Delta$. Hence it is also a morphism of left $\mathbf{k}$-modules. By hypothesis, $\mathbf{u} \cdot \mathbf{h}+\mathbf{h} \cdot \mathbf{v}$ is a left $\mathbf{k}$-submodule of $\mathbf{h} \cdot \mathbf{h}$. Hence, $\mathbf{u} \wedge \mathbf{v}=\Delta^{-1}(\mathbf{u} \cdot \mathbf{h}+\mathbf{h} \cdot \mathbf{v})$ is a left $\mathbf{k}$-submodule of $\mathbf{h}$.

Lemma 11. Let $\mathbf{h}$ be a Hopf monoid in species and $\mathbf{k}$ a Hopf submonoid. Let $\mathbf{c}$ be a subcomonoid of $\mathbf{h}$ and a left $\mathbf{k}$-submodule of $\mathbf{h}$. Then $(\mathbf{k} \wedge \mathbf{c}) / \mathbf{c}$ is a left $\mathbf{k}$-Hopf module.

Proof. By Lemma 10, $\mathbf{k} \wedge \mathbf{c}$ is a left $\mathbf{k}$-submodule of $\mathbf{h}$. Therefore, the quotient $(\mathbf{k} \wedge \mathbf{c}) / \mathbf{c}$ by the left $\mathbf{k}$-submodule $\mathbf{c}$ is a left $\mathbf{k}$-module.

We next argue that $(\mathbf{k} \wedge \mathbf{c}) / \mathbf{c}$ is a $\mathbf{k}$-comodule. Consider the composite

$$
\mathrm{k} \wedge \mathrm{c} \stackrel{\Delta}{\rightarrow} \mathbf{k} \cdot(\mathrm{k} \wedge \mathrm{c})+(\mathrm{k} \wedge \mathrm{c}) \cdot \mathrm{c} \rightarrow \mathrm{k} \cdot(\mathrm{k} \wedge \mathrm{c}) / \mathrm{c},
$$

where the first map is granted by Lemma 9 and the second is the projection modulo $\mathbf{c}$ on the second coordinate. Since $\mathbf{c}$ is a subcomonoid, the composite factors through $\mathbf{c}$ and induces

$$
(\mathrm{k} \wedge \mathrm{c}) / \mathrm{c} \rightarrow \mathrm{k} \cdot(\mathrm{k} \wedge \mathrm{c}) / \mathrm{c}
$$

This defines a left $\mathbf{k}$-comodule structure on $(\mathbf{k} \wedge \mathbf{c}) / \mathbf{c}$.

Finally, the compatibility between the module and comodule structures on $(\mathbf{k} \wedge \mathbf{c}) / \mathbf{c}$ is inherited from the compatibility between the product and coproduct of $\mathbf{h}$.

We are nearly ready for the proof of the main result. First, recall the coradical filtration of a connected comonoid in species [2, Section 8.10]. Given a connected comonoid $\mathbf{c}$, define subspecies $\mathbf{c}_{(n)}$ by

$$
\mathbf{c}_{(0)}=\mathbf{1} \text { and } \quad \mathbf{c}_{(n)}=\mathbf{c}_{(0)} \wedge \mathbf{c}_{(n-1)} \text { for all } n \geq 1 .
$$

We then have

$$
\mathbf{c}_{(0)} \subseteq \mathbf{c}_{(1)} \subseteq \cdots \subseteq \mathbf{c}_{(n)} \subseteq \cdots \mathbf{c} \quad \text { and } \quad \mathbf{c}=\bigcup_{n \geq 0} \mathbf{c}_{(n)}
$$

Proof of Theorem 7 . We show that there is a species $\mathbf{q}$ such that $\mathbf{h} \cong \mathbf{k} \cdot \mathbf{q}$ as left $\mathbf{k}$-modules. As in the proof of Theorem 3, it then follows that $\mathbf{q} \cong \mathbf{h} / \mathbf{k}_{+} \mathbf{h}$.

Define a sequence $\mathbf{k}^{(n)}$ of subspecies of $\mathbf{h}$ by

$$
\mathbf{k}^{(0)}=\mathbf{k} \quad \text { and } \quad \mathbf{k}^{(n)}=\mathbf{k} \wedge \mathbf{k}^{(n-1)} \text { for all } n \geq 1 .
$$


Each $\mathbf{k}^{(n)}$ is a subcomonoid and a left $\mathbf{k}$-submodule of $\mathbf{h}$. This follows from Lemmas 9 and 10 by induction on $n$. Then Lemma 11 provides a left $\mathbf{k}$-Hopf module structure on the quotient species $\mathbf{k}^{(n)} / \mathbf{k}^{(n-1)}$ for all $n \geq 1$. Hence $\mathbf{k}^{(n)} / \mathbf{k}^{(n-1)}$ is a free left $\mathbf{k}$-module, by Proposition 8 .

We claim that there exists a sequence of species $\mathbf{q}_{n}$ such that

$$
\mathbf{k}^{(n)} \cong \mathbf{k} \cdot \mathbf{q}_{n}
$$

as left $\mathbf{k}$-modules for all $n \geq 0$; that is, each $\mathbf{k}^{(n)}$ is a free left $\mathbf{k}$-module. Moreover, we claim that the $\mathbf{q}_{n}$ can be chosen so that

$$
\mathbf{q}_{0} \subseteq \mathbf{q}_{1} \subseteq \cdots \subseteq \mathbf{q}_{n} \subseteq \cdots
$$

and the above isomorphisms are compatible with the inclusions $\mathbf{q}_{n-1} \subseteq \mathbf{q}_{n}$ and $\mathbf{k}^{(n-1)} \subseteq$ $\mathbf{k}^{(n)}$.

We prove the claims by induction on $n \geq 0$. We start by letting $\mathbf{q}_{0}=\mathbf{1}$. For $n \geq 1$, we have

$$
\mathbf{k}^{(n-1)} \cong \mathbf{k} \cdot \mathbf{q}_{n-1} \text { and } \mathbf{k}^{(n)} / \mathbf{k}^{(n-1)} \cong \mathbf{k} \cdot \mathbf{q}_{n}^{\prime}
$$

for some species $\mathbf{q}_{n}^{\prime}$ (the former by induction hypothesis and the latter by the above argument). Let

$$
\mathbf{q}_{n}=\mathbf{q}_{n-1} \oplus \mathbf{q}_{n}^{\prime} .
$$

By choosing an arbitrary $\mathbf{k}$-module section of the map $\mathbf{k}^{(n)} \rightarrow \mathbf{k}^{(n)} / \mathbf{k}^{(n-1)} \cong \mathbf{k} \cdot \mathbf{q}_{n}^{\prime}$ (possible by freeness), we obtain an isomorphism

$$
\mathbf{k}^{(n)} \cong \mathbf{k} \cdot \mathbf{q}_{n}
$$

extending the isomorphism $\mathbf{k}^{(n-1)} \cong \mathbf{k} \cdot \mathbf{q}_{n-1}$. This proves the claims.

We utilize the coradical filtration of $\mathbf{h}$ to finish the proof. Since $\mathbf{h}$ is connected, $\mathbf{h}_{(0)}=\mathbf{1} \subseteq \mathbf{k}=\mathbf{k}^{(0)}$, and by induction,

$$
\mathbf{h}_{(n)} \subseteq \mathbf{k}^{(n)} \quad \text { for all } n \geq 0
$$

Hence,

$$
\mathbf{h}=\bigcup_{n \geq 0} \mathbf{h}_{(n)}=\bigcup_{n \geq 0} \mathbf{k}^{(n)} \cong \bigcup_{n \geq 0} \mathbf{k} \cdot \mathbf{q}_{n} \cong \mathbf{k} \cdot \mathbf{q} \text { where } \mathbf{q}=\bigcup_{n \geq 0} \mathbf{q}_{n} .
$$

Thus $\mathbf{h}$ is free as a left $\mathbf{k}$-module.

Let $\pi: \mathbf{h} \rightarrow \mathbf{k}$ be a morphism of Hopf monoids. The right Hopf kernel of $\pi$ is the species defined by

$$
\operatorname{Hker}(\pi)=\operatorname{ker}\left(\mathbf{h} \stackrel{\Delta}{\rightarrow} \mathbf{h} \cdot \mathbf{h} \stackrel{\pi_{+} \cdot \text { id }}{\longrightarrow} \mathbf{k}_{+} \cdot \mathbf{h}\right),
$$

where $\pi_{+}: \mathbf{h} \rightarrow \mathbf{k}_{+}$is $\pi$ followed by the canonical projection $\mathbf{k} \rightarrow \mathbf{k}_{+}$.

For the following result, we employ duality for Hopf monoids [2, Section 8.6.2]. (We assume all species are finite dimensional.)

Theorem 12. Let $\mathbf{h}$ be a connected Hopf monoid in the category of species and $\mathbf{k}$ a quotient Hopf monoid via a morphism $\pi: \mathbf{h} \rightarrow \mathbf{k}$. Then $\mathbf{h}$ is a cofree left $\mathbf{k}$-comodule. Moreover,

as left $\mathbf{k}$-comodules (and as species).

$$
\mathbf{h} \cong \mathbf{k} \cdot \operatorname{Hker}(\pi)
$$


Proof. By duality, $\mathbf{k}^{*}$ is a Hopf submonoid of $\mathbf{h}^{*}$, so $\mathbf{h}^{*} \cong \mathbf{k}^{*} \cdot\left(\mathbf{h}^{*} / \mathbf{k}_{+}^{*} \mathbf{h}^{*}\right)$ by Theorem 7 . Dualizing again we obtain the result, since

$$
\mathbf{h}^{*} / \mathbf{k}_{+}^{*} \mathbf{h}^{*}=\operatorname{coker}\left(\mathbf{k}_{+}^{*} \cdot \mathbf{h}^{*} \stackrel{\pi_{+}^{*} \cdot \mathrm{id}}{\longrightarrow} \mathbf{h}^{*} \cdot \mathbf{h}^{*} \stackrel{\Delta^{*}}{\longrightarrow} \mathbf{h}^{*}\right) .
$$

\section{Applications AND EXAmples}

3.1. A test for Hopf submonoids. Two invariants associated to a (finite dimensional) species $\mathbf{q}$ are the exponential generating series $\mathcal{E}_{\mathbf{q}}(x)$ and the type generating series $\mathcal{T}_{\mathbf{q}}(x)$. They are given by

$$
\mathcal{E}_{\mathbf{q}}(x)=\sum_{n \geq 0} \operatorname{dim} \mathbf{q}[n] \frac{x^{n}}{n !} \quad \text { and } \quad \mathcal{T}_{\mathbf{q}}(x)=\sum_{n \geq 0} \operatorname{dim} \mathbf{q}[n]_{S_{n}} x^{n},
$$

where

$$
\mathbf{q}[n]_{S_{n}}=\mathbf{q}[n] / \mathbb{k}\left\{v-\sigma \cdot v \mid v \in \mathbf{q}[n], \sigma \in S_{n}\right\} .
$$

Both are specializations of the cycle index series $\mathcal{Z}_{\mathbf{q}}\left(x_{1}, x_{2}, \ldots\right)$; see [4, Section 1.2] for the definition. Specifically,

$$
\mathcal{E}_{\mathbf{q}}(x)=\mathcal{Z}_{\mathbf{q}}(x, 0,0, \ldots) \text { and } \mathcal{T}_{\mathbf{q}}(x)=\mathcal{Z}_{\mathbf{q}}\left(x, x^{2}, x^{3}, \ldots\right) .
$$

The cycle index series is multiplicative under Cauchy product [4, Section 1.3]: if $\mathbf{h}=\mathbf{k} \cdot \mathbf{q}$, then $\mathcal{Z}_{\mathbf{h}}\left(x_{1}, x_{2}, \ldots\right)=\mathcal{Z}_{\mathbf{k}}\left(x_{1}, x_{2}, \ldots\right) \mathcal{Z}_{\mathbf{q}}\left(x_{1}, x_{2}, \ldots\right)$. By specialization, the same is true for the exponential and type generating series.

Let $\mathbb{Q}_{\geq 0}$ denote the nonnegative rational numbers. An immediate consequence of Theorems 7 and 12 is the following.

Corollary 13. Let $\mathbf{h}$ and $\mathbf{k}$ be connected Hopf monoids in species. Suppose $\mathbf{k}$ is either a Hopf submonoid or a quotient Hopf monoid of $\mathbf{h}$. Then the quotient of cycle index series $\mathcal{Z}_{\mathbf{h}}\left(x_{1}, x_{2}, \ldots\right) / \mathcal{Z}_{\mathbf{k}}\left(x_{1}, x_{2}, \ldots\right)$ is nonnegative, i.e., belongs to $\mathbb{Q}_{\geq 0} \llbracket x_{1}, x_{2}, \ldots \rrbracket$. In particular, the quotients $\mathcal{E}_{\mathbf{h}}(x) / \mathcal{E}_{\mathbf{k}}(x)$ and $\mathcal{T}_{\mathbf{h}}(x) / \mathcal{T}_{\mathbf{k}}(x)$ are also nonnegative.

Given a connected Hopf monoid $\mathbf{h}$ in species, we may use Corollary 13 to determine if a given species $\mathbf{k}$ may be a Hopf submonoid (or a quotient Hopf monoid).

Example 14. A partition of a set $I$ is an unordered collection of disjoint nonempty subsets of $I$ whose union is $I$. The notation $a b . c$ is shorthand for the partition $\{\{a, b\},\{c\}\}$ of $\{a, b, c\}$.

Let $\Pi$ be the species of set partitions, i.e., $\Pi[I]$ is the vector space with basis the set of all partitions of $I$. Let $\boldsymbol{\Pi}^{\prime}$ denote the subspecies linearly spanned by set partitions with distinct block sizes. For example,

$$
\Pi[a, b, c]=\mathbb{k}\{a b c, a \cdot b c, a b . c, a \cdot b c, a \cdot b \cdot c\} \quad \text { and } \quad \Pi^{\prime}[a, b, c]=\mathbb{k}\{a b c, a \cdot b c, a b . c, a \cdot b c\} .
$$

The sequences $(\operatorname{dim} \Pi[n])_{n \geq 0}$ and $\left(\operatorname{dim} \boldsymbol{\Pi}^{\prime}[n]\right)_{n \geq 0}$ appear in [29] as A000110 and A007837, respectively. We have

$$
\mathcal{E}_{\boldsymbol{\Pi}}(x)=\exp (\exp (x)-1)=1+x+x^{2}+\frac{5}{6} x^{3}+\frac{5}{8} x^{4}+\cdots
$$

and

$$
\mathcal{E}_{\Pi^{\prime}}(x)=\prod_{n \geq 1}\left(1+\frac{x^{n}}{n !}\right)=1+x+\frac{1}{2} x^{2}+\frac{2}{3} x^{3}+\frac{5}{24} x^{4}+\cdots
$$


A Hopf monoid structure on $\boldsymbol{\Pi}$ is defined in [2, Section 12.6]. There are many linear bases of $\Pi$ indexed by set partitions, and many ways to embed $\boldsymbol{\Pi}^{\prime}$ as a subspecies of $\boldsymbol{\Pi}$. Is it possible to embed $\boldsymbol{\Pi}^{\prime}$ as a Hopf submonoid of $\boldsymbol{\Pi}$ ? We have

$$
\mathcal{E}_{\boldsymbol{\Pi}}(x) / \mathcal{E}_{\boldsymbol{\Pi}^{\prime}}(x)=1+\frac{1}{2} x^{2}-\frac{1}{3} x^{3}+\frac{1}{2} x^{4}-\frac{11}{30} x^{5}+\cdots,
$$

so the answer is negative by Corollary 13. In fact, it is not possible to embed $\boldsymbol{\Pi}^{\prime}$ as a Hopf submonoid of $\boldsymbol{\Pi}$ for any potential Hopf monoid structure on $\boldsymbol{\Pi}$.

We remark that the type generating series quotient for the pair of species in Example 14 is nonnegative:

$$
\begin{aligned}
\mathcal{T}_{\Pi}(x) & =1+x+2 x^{2}+3 x^{3}+5 x^{4}+7 x^{5}+11 x^{6}+15 x^{7}+\cdots, \\
\mathcal{T}_{\Pi^{\prime}}(x) & =1+x+x^{2}+2 x^{3}+2 x^{4}+3 x^{5}+4 x^{6}+5 x^{7}+\cdots, \\
\mathcal{T}_{\Pi}(x) / \mathcal{T}_{\Pi^{\prime}}(x) & =1+x^{2}+2 x^{4}+3 x^{6}+5 x^{8}+7 x^{10}+\cdots .
\end{aligned}
$$

This can be understood by appealing to the Hopf algebra Sym of symmetric functions. A basis for its homogenous component of degree $n$ is indexed by integer partitions of $n$, so $\mathcal{O}_{\text {Sym }}(x)=\mathcal{T}_{\Pi}(x)$. Moreover, $\mathcal{T}_{\Pi^{\prime}}(x)$ enumerates the integer partitions with odd part sizes and Sym does indeed contain a Hopf subalgebra with this Poincaré series. It is the algebra of Schur $Q$-functions. See [15, III.8]. Thus $\mathcal{T}_{\Pi}(x) / \mathcal{T}_{\Pi^{\prime}}(x)$ is nonnegative by Corollary 4.

3.2. Tests for Hopf monoids. Let $\left(a_{n}\right)_{n \geq 0}$ be a sequence of nonnegative integers with $a_{0}=1$. Does there exist a connected Hopf monoid $\mathbf{h}$ with $\operatorname{dim} \mathbf{h}[n]=a_{n}$ for all $n$ ? The next result provides conditions that the sequence $\left(a_{n}\right)_{n \geq 0}$ must satisfy in order for this to be the case. The proof makes use of the Hadamard product of Hopf monoids [2, Sections 8.1 and 8.13]. If $\mathbf{h}$ and $\mathbf{k}$ are Hopf monoids, so is $\mathbf{h} \times \mathbf{k}$, with $(\mathbf{h} \times \mathbf{k})[I]=\mathbf{h}[I] \otimes \mathbf{k}[I]$ for each finite set $I$. The exponential species $\mathbf{E}$ is the unit element for the Hadamard product.

Corollary 15 (The (ord/exp)-test). For any connected Hopf monoid in species $\mathbf{h}$,

$$
\left(\sum_{n \geq 0} \operatorname{dim} \mathbf{h}[n] x^{n}\right) /\left(\sum_{n \geq 0} \frac{\operatorname{dim} \mathbf{h}[n]}{n !} x^{n}\right) \in \mathbb{Q}_{\geq 0} \llbracket x \rrbracket .
$$

Proof. Consider the canonical morphism of Hopf monoids $\mathbf{L} \rightarrow \mathbf{E}$ [2, Section 8.5]; it maps any linear order $\ell \in \mathbf{L}[I]$ to the basis element $*_{I} \in \mathbf{E}[I]$. The Hadamard product then yields a morphism of Hopf monoids

$$
\mathbf{L} \times \mathbf{h} \rightarrow \mathbf{E} \times \mathbf{h} \cong \mathbf{h} .
$$

By Corollary $13, \mathcal{E}_{\mathbf{L} \times \mathbf{h}}(x) / \mathcal{E}_{\mathbf{h}}(x) \in \mathbb{Q}_{\geq 0} \llbracket x \rrbracket$. Since $\mathcal{E}_{\mathbf{L} \times \mathbf{h}}(x)=\sum_{n \geq 0} \operatorname{dim} \mathbf{h}[n] x^{n}$, the result follows.

Let $a_{n}=\operatorname{dim} \mathbf{h}[n]$. Corollary 15 states that the ratio of the ordinary to the exponential generating function of the sequence $\left(a_{n}\right)_{n \geq 0}$ must be nonnegative. This translates into a sequence of polynomial inequalities, the first of which are as follows:

$$
5 a_{3} \geq 3 a_{2} a_{1}, \quad 23 a_{4}+12 a_{2} a_{1}^{2} \geq 20 a_{3} a_{1}+6 a_{2}^{2} .
$$


In particular, not every nonnegative sequence arises as the dimension sequence of a Hopf monoid.

The following test is of a similar nature, but involves the type instead of the exponential generating function. The conditions then depend not just on the dimension sequence of $\mathbf{h}$, but also on its species structure.

Corollary 16 (The (ord/type)-test). For any connected Hopf monoid in species $\mathbf{h}$,

$$
\left(\sum_{n \geq 0} \operatorname{dim} \mathbf{h}[n] x^{n}\right) /\left(\sum_{n \geq 0} \operatorname{dim} \mathbf{h}[n]_{S_{n}} x^{n}\right) \in \mathbb{N} \llbracket x \rrbracket .
$$

Proof. We argue as in the proof of Corollary 15, using type generating functions instead. Since we have $\mathcal{T}_{\mathbf{L} \times \mathbf{h}}(x)=\sum_{n \geq 0} \operatorname{dim} \mathbf{h}[n] x^{n}$, the result follows.

Remark. The previous result may also be derived as follows. According to [2, Chapter 15], associated to the Hopf monoid $\mathbf{h}$ there are two graded Hopf algebras $\mathcal{K}(\mathbf{h})$ and $\overline{\mathcal{K}}(\mathbf{h})$, as well as a surjective morphism

$$
\mathcal{K}(\mathbf{h}) \rightarrow \overline{\mathcal{K}}(\mathbf{h}) .
$$

Moreover, the Poincaré series for these Hopf algebras are

$$
\mathcal{O}_{\mathcal{K}(\mathbf{h})}(x)=\sum_{n \geq 0} \operatorname{dim} \mathbf{h}[n] x^{n} \quad \text { and } \quad \mathcal{O}_{\overline{\mathcal{K}}(\mathbf{h})}(x)=\sum_{n \geq 0} \operatorname{dim} \mathbf{h}[n]_{S_{n}} x^{n} .
$$

Corollary 16 now follows from (the dual form of) Corollary 4.

3.3. Additional tests for Hopf monoids. The method of Section 3.2 can be applied in multiple situations in order to deduce additional inequalities that the dimension sequence of a connected Hopf monoid must satisfy. We illustrate this next.

Let $k$ be a fixed nonnegative integer. Let $\mathbf{E}^{\cdot k}$ denote the $k$-th Cauchy power of the exponential species $\mathbf{E}$. The space $\mathbf{E}^{\cdot k}[I]$ has a basis consisting of functions $f: I \rightarrow[k]$. The species $\mathbf{E}^{\cdot k}$ carries a Hopf monoid structure [2, Examples 8.17 and 8.18] and any fixed inclusion $[k] \hookrightarrow[k+1]$ gives rise to an injective morphism of Hopf monoids $\mathbf{E}^{\cdot k} \hookrightarrow$ $\mathbf{E}^{\cdot(k+1)}$. Employing the Hadamard product as in Section 3.2, we obtain an injective morphism of Hopf monoids

$$
\mathbf{E}^{\cdot k} \times \mathbf{h} \hookrightarrow \mathbf{E}^{\cdot(k+1)} \times \mathbf{h}
$$

where $\mathbf{h}$ is an arbitrary connected Hopf monoid. From the nonnegativity of the first coefficients of $\mathcal{E}_{\mathbf{E}^{(k+1)} \times \mathbf{h}}(x) / \mathcal{E}_{\mathbf{E}^{* k} \times \mathbf{h}}(x)$ we obtain

$$
(2 k+1) a_{2} \geq 2 k a_{1}^{2} \quad \text { and } \quad\left(3 k^{2}+3 k+1\right) a_{3} \geq 3\left(3 k^{2}+k\right) a_{2} a_{1}-6 k^{2} a_{1}^{3} .
$$

These inequalities hold for every $k \in \mathbb{N}$. Letting $k \rightarrow \infty$ we deduce

$$
a_{2} \geq a_{1}^{2} \quad \text { and } \quad a_{3} \geq 3 a_{2} a_{1}-2 a_{1}^{3} .
$$

Example 17. Consider the species e of elements. The set $I$ is a basis of the space $\mathbf{e}[I]$, so the dimension sequence of $\mathbf{e}$ is $a_{n}=n$. This sequence does not satisfy the second inequality in (3). Therefore, the species e does not carry any Hopf monoid structure. 
3.4. A test for Hopf monoids over E. Our next result is a necessary condition for a Hopf monoid in species to contain or surject onto the exponential species $\mathbf{E}$.

Given a sequence $\left(a_{n}\right)_{n \geq 0}$, its binomial transform $\left(b_{n}\right)_{n \geq 0}$ is defined by

$$
b_{n}:=\sum_{i=0}^{n}\left(\begin{array}{l}
n \\
i
\end{array}\right)(-1)^{i} a_{n-i} .
$$

Corollary 18 (The E-test). Suppose $\mathbf{h}$ is a connected Hopf monoid that either contains the species $\mathbf{E}$ or surjects onto $\mathbf{E}$ (in both cases as a Hopf monoid). Let $a_{n}=\operatorname{dim} \mathbf{h}[n]$ and $\bar{a}_{n}=\operatorname{dim} \mathbf{h}[n]_{S_{n}}$. Then the binomial transform of $\left(a_{n}\right)_{n \geq 0}$ must be nonnegative and $\left(\bar{a}_{n}\right)_{n \geq 0}$ must be nondecreasing.

More plainly, in this setting, we must have the following inequalities:

$$
a_{1} \geq a_{0}, \quad a_{2} \geq 2 a_{1}-a_{0}, \quad a_{3} \geq 3 a_{2}-3 a_{1}+a_{0}, \quad \ldots
$$

and $\bar{a}_{n} \geq \bar{a}_{n-1}$ for all $n \geq 1$.

Proof. By Corollary 13, the quotient $\mathcal{E}_{\mathbf{h}}(x) / \mathcal{E}_{\mathbf{E}}(x)$ is nonnegative. But $\mathcal{E}_{\mathbf{E}}(x)=\exp (x)$, so the quotient is given by

$$
b_{0}+b_{1} x+b_{2} \frac{x^{2}}{2}+b_{3} \frac{x^{3}}{3 !}+\cdots
$$

where $\left(b_{n}\right)_{n \geq 0}$ is the binomial transform of $\left(a_{n}\right)_{n \geq 0}$. Replacing exponential for type generating functions yields the result for $\left(\bar{a}_{n}\right)_{n \geq 0}$, since $\mathcal{T}_{\mathbf{E}}(x)=\frac{1}{1-x}$.

Remark. Myhill's theory of combinatorial functions [6, 19] provides necessary and sufficient conditions that a sequence $\left(a_{n}\right)_{n \geq 0}$ must satisfy in order for its binomial transform to be nonnegative: the sequence must arise from a particular type of operator defined on finite sets. Work of Menni [17] expands on this from a categorical perspective. It would be interesting to relate these ideas to the ones of this paper.

We make a further remark regarding connected linearized Hopf monoids. These are Hopf monoids of a set theoretic nature. See [2, Section 8.7] for details. Briefly, there are set maps $\mu_{S, T}: \mathrm{H}[S] \times \mathrm{H}[T] \rightarrow \mathrm{H}[I]$ and $\Delta_{S, T}: \mathrm{H}[I] \rightarrow \mathrm{H}[S] \times \mathrm{H}[T]$ which produce a Hopf monoid in (vector) species when the set species $\mathrm{H}$ is linearized. It follows that if $\mathbf{h}$ is a linearized Hopf monoid other than the trivial Hopf monoid $\mathbf{1}$, then there is a morphism of Hopf monoids from $\mathbf{h}$ onto E. Thus, Corollary 18 provides a test for existence of a linearized Hopf monoid structure on $\mathbf{h}$.

Example 19. We return to the species $\boldsymbol{\Pi}^{\prime}$ of set partitions into distinct block sizes. We might ask if this can be made into a linearized Hopf monoid in some way (after Example 14, this would not be as a Hopf submonoid of $\boldsymbol{\Pi}$ ). With $a_{n}$ and $b_{n}$ as above, we have:

$$
\begin{aligned}
& \left(a_{n}\right)_{n \geq 0}=1,1,1,4,5,16,82,169,541, \ldots \\
& \left(b_{n}\right)_{n \geq 0}=1,0,0,3,-8,25,-9,-119,736, \ldots
\end{aligned}
$$

Thus $\boldsymbol{\Pi}^{\prime}$ fails the $\mathbf{E}$-test and the answer to the above question is negative. 
3.5. A test for Hopf monoids over $\mathbf{L}$. Let $\mathbf{h}$ be a connected Hopf monoid in species. Let $a_{n}=\operatorname{dim} \mathbf{h}[n]$ and $\bar{a}_{n}=\operatorname{dim} \mathbf{h}[n]_{S_{n}}$. Note that the analogous integers for the species $\mathbf{L}$ of linear orders are $b_{n}=n$ ! and $\bar{b}_{n}=1$. Now suppose that $\mathbf{h}$ contains $\mathbf{L}$ or surjects onto $\mathbf{L}$ as a Hopf monoid. An obvious necessary condition for this situation is that $a_{n} \geq n !$ and $\bar{a}_{n} \geq 1$. Our next result provides stronger conditions.

Corollary 20 (The L-test). Suppose $\mathbf{h}$ is a connected Hopf monoid that either contains the species $\mathbf{L}$ or surjects onto $\mathbf{L}$ (in both cases as a Hopf monoid). If $a_{n}=\operatorname{dim} \mathbf{h}[n]$ and $\bar{a}_{n}=\operatorname{dim} \mathbf{h}[n]_{S_{n}}$, then

$$
a_{n} \geq n a_{n-1} \quad \text { and } \quad \bar{a}_{n} \geq \bar{a}_{n-1} \quad(\forall n \geq 1) .
$$

Proof. It follows from Corollary 13 that both $\mathcal{E}_{\mathbf{h}}(x) / \mathcal{E}_{\mathbf{L}}(x)$ and $\mathcal{T}_{\mathbf{h}}(x) / \mathcal{T}_{\mathbf{L}}(x)$ are nonnegative. These yield the first and second set of inequalities, respectively.

Before giving an application of the corollary, we introduce a new Hopf monoid in species. A composition of a set $I$ is an ordered collection of disjoint nonempty subsets of $I$ whose union is $I$. The notation $a b \mid c$ is shorthand for the composition $(\{a, b\},\{c\})$ of $\{a, b, c\}$.

Let $\mathbf{P a l}$ denote the species of set compositions whose sequence of block sizes is palindromic. So, for instance,

$$
\operatorname{Pal}[a, b]=\mathbb{k}\{a b, a|b, b| a\}
$$

and

$$
\operatorname{Pal}[a, b, c, d, e]=\mathbb{k}\{a b c d e, a|b c d| e, a b|c| d e, a|b| c|d| e, \ldots\} .
$$

The latter space has dimension $171=1+5\left(\begin{array}{l}4 \\ 3\end{array}\right)+\left(\begin{array}{l}5 \\ 2\end{array}\right) 3+5$ ! while $\operatorname{dim} \operatorname{Pal}[5]_{S_{5}}=4$ (accounting for the four types of palindromic set compositions shown above).

Given a palindromic set composition $F=F_{1}|\cdots| F_{r}$, we view it as a triple $F=$ $\left(F^{-}, F^{0}, F^{+}\right)$, where $F^{-}$is the initial sequence of blocks, $F^{0}$ is the central block if this exists (if the number of blocks is odd) and otherwise it is the empty set, and $F^{+}$is the final sequence of blocks. That is,

$$
F^{-}=F_{1}|\cdots| F_{\lfloor r / 2\rfloor}, \quad F^{0}=\left\{\begin{array}{ll}
F_{\lfloor r / 2\rfloor+1} & \text { if } r \text { is odd, } \\
\emptyset & \text { if } r \text { is even, }
\end{array} \quad F^{+}=F_{\lceil r / 2+1\rceil}|\cdots| F_{r} .\right.
$$

Given $S \subseteq I$, let

$$
\left.F\right|_{S}:=F_{1} \cap S\left|F_{2} \cap S\right| \cdots \mid F_{r} \cap S,
$$

where empty intersections are deleted. Then $\left.F\right|_{S}$ is a composition of $S$. Let us say that $S$ is admissible for $F$ if

$$
\#\left(F_{i} \cap S\right)=\#\left(F_{r+1-i} \cap S\right) \text { for all } i=1, \ldots, r .
$$

In this case, both $\left.F\right|_{S}$ and $\left.F\right|_{I \backslash S}$ are palindromic.

We employ the above notation to define product and coproduct operations on Pal. Fix a decomposition $I=S \sqcup T$.

Product. Given palindromic set compositions $F \in \mathbf{P a l}[S]$ and $G \in \mathbf{P a l}[T]$, we put

$$
\mu_{S, T}(F \otimes G):=\left(F^{-}\left|G^{-}, F^{0} \cup G^{0}, G^{+}\right| F^{+}\right) .
$$


In other words, we concatenate the initial sequences of blocks of $F$ and $G$ in that order, merge their central blocks, and concatenate their final sequences in the opposite order. The result is a palindromic composition of $I$. For example, with $S=\{a, b\}$ and $T=\{c, d, e, f\}$,

$$
(a \mid b) \otimes(c|d e| f) \mapsto a|c| d e|f| b .
$$

Coproduct. Given a palindromic set composition $F \in \mathbf{P a l}[I]$, we put

$$
\Delta_{S, T}(F):= \begin{cases}\left.\left.F\right|_{S} \otimes F\right|_{T} & \text { if } S \text { is admissible for } F \\ 0 & \text { otherwise. }\end{cases}
$$

For example, with $S$ and $T$ as above,

$$
a d|b| e \mid c f \mapsto 0 \quad \text { and } \quad e|a b c d| f \mapsto(a b) \otimes(e|c d| f) \text {. }
$$

These operations endow Pal with the structure of Hopf monoid, as may be easily checked.

Example 21. A linear order may be seen as a palindromic set composition (with singleton blocks). Both Hopf monoids Pal and $\mathbf{L}$ are cocommutative and not commutative. We may then ask if Pal contains (or surjects onto) $\mathbf{L}$ as a Hopf monoid.

Writing $a_{n}=\operatorname{dim} \operatorname{Pal}[n]$, we have:

$$
\left(a_{n}\right)_{n \geq 0}=1,1,3,7,43,171,1581,8793,108347, \ldots
$$

However,

$$
\left(a_{n}-n a_{n-1}\right)_{n \geq 1}=0,1,-2,15,-44,555,-2274,38003, \ldots,
$$

so Pal fails the $\mathbf{L}$-test and the answer to the above question is negative.

3.6. Examples of nonnegative quotients. We comment on a few examples where the quotient power series $\mathcal{E}_{\mathbf{h}}(x) / \mathcal{E}_{\mathbf{k}}(x)$ is not only nonnegative but is known to have a combinatorial interpretation as a generating function.

Example 22. Consider the Hopf monoid $\boldsymbol{\Pi}$ of set partitions. It contains $\mathbf{E}$ as a Hopf submonoid via the map that sends $*_{I}$ to the partition of $I$ into singletons. We have

$$
\mathcal{E}_{\boldsymbol{\Pi}}(x) / \mathcal{E}_{\mathbf{E}}(x)=\exp (\exp (x)-x-1),
$$

which is the exponential generating function for the number of set partitions into blocks of size strictly bigger than 1 . This fact may also be understood with the aid of Theorem 7 , as follows.

Given $I=S \sqcup T$, the product of a partition $\pi \in \Pi[S]$ and a partition $\rho \in \Pi[T]$ is the partition $\pi \cdot \rho \in \Pi[I]$ each of whose blocks is either a block of $\pi$ or a block of $\rho$. (In the notation of [2, Section 12.6], we are employing the $h$-basis of $\boldsymbol{\Pi}$.) Now, the $I$-component of the right ideal $\mathbf{E}_{+} \boldsymbol{\Pi}$ is linearly spanned by elements of the form $*_{S} \cdot \pi$ where $I=S \sqcup T$ and $\pi$ is a partition of $T$. Then, since $*_{S}=*_{\{i\}} \cdot *_{S \backslash\{i\}}$ (for any $\left.i \in S\right)$, we have that $\mathbf{E}_{+} \boldsymbol{\Pi}[I]$ is linearly spanned by elements of the form $*_{\{i\}} \cdot \pi$ where $i \in I$ and $\pi$ is a partition of $I \backslash\{i\}$. But the above description of the product shows that these are precisely the partitions with at least one singleton block. 
Example 23. Consider the Hopf monoids $\mathbf{L}$ and $\mathbf{E}$ and the surjective morphism $\pi$ : $\mathbf{L} \rightarrow \mathbf{E}$ (as in the proof of Corollary 15). We have

$$
\mathcal{E}_{\mathbf{L}}(x)=\frac{1}{1-x} \quad \text { and } \quad \mathcal{E}_{\mathbf{E}}(x)=\exp (x) .
$$

It is well-known [4, Example 1.3.9] that

$$
\frac{\exp (-x)}{1-x}=\sum_{n \geq 0} \frac{d_{n}}{n !} x^{n}
$$

where $d_{n}$ is the number of derangements of $[n]$. Together with Theorem 12 , this suggests the existence of a basis for the Hopf kernel of $\pi$ indexed by derangements. We construct such a basis and expand on this discussion in Section 5.3.

Example 24. Let $\boldsymbol{\Sigma}$ be the Hopf monoid of set compositions defined in [2, Section 12.4]. It contains $\mathbf{L}$ as a Hopf submonoid via the map that views a linear order as a composition into singletons. (In the notation of [2, Section 12.4], we are employing the $H$-basis of $\boldsymbol{\Sigma}$.) This and other morphisms relating $\mathbf{E}, \mathbf{L}, \boldsymbol{\Pi}$ and $\boldsymbol{\Sigma}$, as well as other Hopf monoids, are discussed in [2, Section 12.8].

The sequence $(\operatorname{dim} \Sigma[n])_{n \geq 0}$ is A000670 in [29]. We have

$$
\mathcal{E}_{\boldsymbol{\Sigma}}(x)=\frac{1}{2-\exp (x)} .
$$

Moreover, it is known from [31, Exercise 5.4.(a)] that

$$
\frac{1-x}{2-\exp (x)}=\sum_{n \geq 0} \frac{s_{n}}{n !} x^{n}
$$

where $s_{n}$ is the number of threshold graphs with vertex set $[n]$ and no isolated vertices. Together with Theorem 7 , this suggests the existence of a basis for $\Sigma / \mathbf{L}_{+} \boldsymbol{\Sigma}$ indexed by such graphs. We do not pursue this possibility in this paper.

\section{The dimension Sequence of A COnnected Hopf Monoid}

Let $\mathbf{h}$ be a connected Hopf monoid and $a_{n}=\operatorname{dim} \mathbf{h}[n]$ for $n \in \mathbb{N}$. The results of Sections 3.2 and 3.3, derived from Theorem 7, impose restrictions on the sequence $a_{n}$ in the form of polynomial inequalities. The results of this section are neither weaker nor stronger than those of Section 3, but provide supplementary information on the dimension sequence $a_{n}$. They do not make use of Theorem 7 . In this section, the base field $\mathbb{k}$ is of arbitrary characteristic.

Proposition 25. For any $n, i$ and $j$ such that $n=i+j$,

$$
a_{n} \geq a_{i} a_{j} .
$$

Proof. Since $\mathbf{h}$ is connected, the compatibility axiom for Hopf monoids (diagram (8.18) in [2, Section 8.3.1]) implies that the composite

$$
\mathbf{h}[S] \otimes \mathbf{h}[T] \stackrel{\mu_{S, T}}{\longrightarrow} \mathbf{h}[I] \stackrel{\Delta_{S, T}}{\longrightarrow} \mathbf{h}[S] \otimes \mathbf{h}[T]
$$

is the identity. The result follows by choosing any decomposition $I=S \sqcup T$ with $|I|=n$, $|S|=i$, and $|T|=j$. 
Remark. The second inequality in (3) may be combined with (4) to obtain

$$
a_{3}-a_{2} a_{1} \geq 2 a_{1}\left(a_{2}-a_{1}^{2}\right) \geq 0 .
$$

Considerations of this type show that neither set of inequalities (2), (3) or (4) follows from the others.

As a first consequence of Proposition 25, we derive a result on the growth of the dimension sequence.

Corollary 26. If $a_{1} \geq 1$, then the sequence $a_{n}$ is weakly increasing. If moreover there exists $k \geq 1$ such that

$$
a_{k} \geq 2 \quad \text { and } \quad a_{i} \geq 1 \forall i=0, \ldots, k-1,
$$

then $a_{n}=O\left(2^{n / k}\right)$.

Proof. The first statement follows from $a_{n} \geq a_{1} a_{n-1}$. Now fix $k$ as in the second statement. Given $n \geq k$, write $n=q k+r$ with $q \in \mathbb{N}$ and $0 \leq r \leq k-1$. From (4) we obtain

$$
a_{n} \geq a_{k}^{q} a_{r} \geq 2^{q}=2^{-r / k} 2^{n / k} .
$$

Thus $a_{n}=O\left(2^{n / k}\right)$.

Define the support of $\mathbf{h}$ to be the support of its dimension sequence; namely,

$$
\operatorname{supp}(\mathbf{h})=\left\{n \in \mathbb{N} \mid a_{n} \neq 0\right\} .
$$

We turn to consequences of Proposition 25 on the support.

Corollary 27. The set $\operatorname{supp}(\mathbf{h})$ is a submonoid of $(\mathbb{N},+)$.

Proof. By (co)unitality of $\mathbf{h}, 0 \in \operatorname{supp}(\mathbf{h})$. (In fact, $a_{0}=1$ by connectedness.) By Proposition 25, the set $\operatorname{supp}(\mathbf{h})$ is closed under addition.

We mention that, conversely, given any submonoid $S$ of $(\mathbb{N},+)$, there exists a connected Hopf monoid $\mathbf{h}$ such that $\operatorname{supp}(\mathbf{h})=S$. Indeed, let $\boldsymbol{\Pi}_{S}[I]$ be the space spanned by the set of partitions of $I$ whose block sizes belong to $S \backslash\{0\}$. Then $\Pi_{S}$ is a quotient Hopf monoid of $\boldsymbol{\Pi}$ and $\operatorname{supp}\left(\boldsymbol{\Pi}_{S}\right)=S$. (The former follows from the formulas in $[2$, Section 12.6.2]; we employ the $h$-basis of $\Pi$.)

Example 28. Consider the special case of the previous paragraph in which $S$ is the submonoid of even numbers. Then $\Pi_{S}$ is the species of set partitions into blocks of even size. In particular, $a_{n}=0$ for all odd $n$, so the dimension sequence is neither increasing nor of exponential growth. This example shows that the hypotheses of Corollary 26 cannot be removed.

Corollary 29. The set $\operatorname{supp}(\mathbf{h})$ is either $\{0\}$ or infinite. The set $\mathbb{N} \backslash \operatorname{supp}(\mathbf{h})$ is finite if and only if $\operatorname{gcd}(\operatorname{supp}(\mathbf{h}))=1$.

Proof. These statements hold for all submonoids of $\mathbb{N}$, hence for $\operatorname{supp}(\mathbf{h})$ by Corollary 27. For the second statement, see [26, Lemma 2.1]. 
Remark. We comment on counterparts for connected graded Hopf algebras of the results of this section.

Consider the polynomial Hopf algebra $H=\mathbb{k}\left[x_{1}, \ldots, x_{k}\right]$, in which the generators $x_{i}$ are primitive and of degree 1 . The dimension sequence is $a_{n}=\left(\begin{array}{c}n+k-1 \\ k-1\end{array}\right)$. In contrast to Corollary 26, this sequence is polynomial even if $a_{1}>1$. It follows that Proposition 25 has no counterpart for connected graded Hopf algebras $H$, and that the multiplication map $H_{i} \otimes H_{j} \rightarrow H_{i+j}$ is not injective in general.

Corollaries 27 and 29 fail for connected Hopf algebras over a field of positive characteristic. In characteristic $p$, a counterexample is provided by $H=\mathbb{k}[x] /\left(x^{p}\right)$ with $x$ primitive and of degree 1.

On the other hand, if the field characteristic is 0 , then the set $S=\left\{n \in \mathbb{N} \mid H_{n} \neq 0\right\}$ is a submonoid of $(\mathbb{N},+)$. This follows from the fact that in this case any connected Hopf algebra is a domain. We expand on this point in the Appendix.

\section{Hopf KeRnels FOR COCOMMUTATIVE Hopf MONOIDS}

Hopf kernels enter in the decomposition of Theorem 12 (and in dual form, in Theorem 7). For cocommutative Hopf monoids, Hopf kernels and Lie kernels are closely related, as discussed in this section. We provide a simple result that allows us to describe the Hopf kernel in certain situations and we illustrate it with the case of the canonical morphism $\mathbf{L} \rightarrow \mathbf{E}$.

5.1. Hopf and Lie kernels. The species $\mathcal{P}(\mathbf{h})$ of primitive elements of a connected Hopf monoid $\mathbf{h}$ is defined by

$$
\mathcal{P}(\mathbf{h})[I]=\{x \in \mathbf{h}[I] \mid \Delta(x)=1 \otimes x+x \otimes 1\}
$$

for each nonempty finite set $I$, and $\mathcal{P}(\mathbf{h})[\emptyset]=0$. Equivalently,

$$
\mathcal{P}(\mathbf{h})[I]=\bigcap_{\substack{S \leq T=I \\ S, T \neq \emptyset}} \operatorname{ker}\left(\Delta_{S, T}: \mathbf{h}[I] \rightarrow \mathbf{h}[S] \otimes \mathbf{h}[T]\right) .
$$

It is a Lie submonoid of $\mathbf{h}$ under the commutator bracket. See [2, Sections 8.10 and 11.9] for more information on primitive elements.

Let $\pi: \mathbf{h} \rightarrow \mathbf{k}$ be a morphism of connected Hopf monoids. It restricts to a morphism of Lie monoids $\mathcal{P}(\mathbf{h}) \rightarrow \mathcal{P}(\mathbf{k})$, which we still denote by $\pi$. We define the Lie kernel of $\pi$ as the species

$$
\operatorname{Lker}(\pi)=\operatorname{ker}(\pi: \mathcal{P}(\mathbf{h}) \rightarrow \mathcal{P}(\mathbf{k})) .
$$

It is a Lie ideal of $\mathcal{P}(\mathbf{h})$. The Hopf kernel Hker $(\pi)$ is defined in (1).

Lemma 30. Let $\pi: \mathbf{h} \rightarrow \mathbf{k}$ be a morphism of connected Hopf monoids. Then

$$
\operatorname{Lker}(\pi) \subseteq \operatorname{Hker}(\pi) .
$$

Proof. Let $x \in \operatorname{Lker}(\pi)$. Then

$$
\left(\pi_{+} \cdot \mathrm{id}\right) \Delta(x)=\left(\pi_{+} \cdot \mathrm{id}\right)(1 \otimes x+x \otimes 1)=0,
$$

since $\pi_{+}(1)=0$ and $\pi(x)=0$. Thus $x \in \operatorname{Hker}(\pi)$.

Lemma 31. Let $\pi: \mathbf{h} \rightarrow \mathbf{k}$ be a morphism of connected Hopf monoids. Then Hker $(\pi)$ is a submonoid of $\mathbf{h}$. 
Proof. By definition,

$$
\operatorname{Hker}(\pi)=\Delta^{-1}(\operatorname{Eq}(\pi \cdot \mathrm{id}, \iota \epsilon \cdot \mathrm{id})),
$$

where $\iota: \mathbf{1} \rightarrow \mathbf{k}$ is the unit of $\mathbf{k}, \epsilon: \mathbf{h} \rightarrow \mathbf{1}$ is the counit of $\mathbf{h}$, and Eq denotes the equalizer of two maps. Since $\pi$ and $\iota \epsilon$ are morphisms of monoids $\mathbf{h} \rightarrow \mathbf{k}$, the above equalizer is a submonoid of $\mathbf{h} \cdot \mathbf{h}$. Since $\Delta$ is a morphism of monoids, $\operatorname{Hker}(\pi)$ is a submonoid of $\mathbf{h}$.

The following result provides the announced connection between Lie and Hopf kernels for cocommutative Hopf monoids. It makes use of the Poincaré-Birkhoff-Witt and Cartier-Milnor-Moore theorems for species, which are discussed in [2, Section 11.9.3].

Proposition 32. Let $\pi: \mathbf{h} \rightarrow \mathbf{k}$ be a surjective morphism of connected cocommutative Hopf monoids. Then Hker $(\pi)$ is the submonoid of $\mathbf{h}$ generated by $\operatorname{Lker}(\pi)$.

Proof. Lemmas 30 and 31 imply one inclusion. To conclude the equality, it suffices to check that the dimensions agree, or equivalently, that the exponential generating series are the same. (We are assuming finite dimensionality throughout.)

First of all, from Theorem 12, we have

$$
\mathcal{E}_{\mathrm{Hker}(\pi)}(x)=\mathcal{E}_{\mathbf{h}}(x) / \mathcal{E}_{\mathbf{k}}(x) .
$$

Now, since $\mathbf{h}$ is cocommutative, we have

$$
\mathbf{h} \cong \mathcal{U}(\mathcal{P}(\mathbf{h})) \cong \mathcal{S}(\mathcal{P}(\mathbf{h}))=\mathbf{E} \circ \mathcal{P}(\mathbf{h})
$$

The first is an isomorphism of Hopf monoids (the Cartier-Milnor-Moore theorem), the second is an isomorphism of comonoids (the Poincaré-Birkhoff-Witt theorem), and the third is the definition of the species underlying $\mathcal{S}(\mathcal{P}(\mathbf{h}))$ [2, Section 11.3]. It follows that

$$
\mathcal{E}_{\mathbf{h}}(x)=\exp \left(\mathcal{E}_{\mathcal{P}(\mathbf{h})}(x)\right)
$$

For the same reason,

$$
\mathcal{E}_{\mathbf{k}}(x)=\exp \left(\mathcal{E}_{\mathcal{P}(\mathbf{k})}(x)\right)
$$

and therefore

$$
\mathcal{E}_{\text {Hker }(\pi)}(x)=\exp \left(\mathcal{E}_{\mathcal{P}(\mathbf{h})}(x)-\mathcal{E}_{\mathcal{P}(\mathbf{k})}(x)\right) .
$$

On the other hand, since the functors $\mathcal{U}$ and $\mathcal{P}$ define an adjoint equivalence, they preserve surjectivity of maps. Thus, the induced map $\pi: \mathcal{P}(\mathbf{h}) \rightarrow \mathcal{P}(\mathbf{k})$ is surjective, and we have an exact sequence

$$
0 \rightarrow \operatorname{Lker}(\pi) \rightarrow \mathcal{P}(\mathbf{h}) \rightarrow \mathcal{P}(\mathbf{k}) \rightarrow 0 .
$$

Hence,

$$
\mathcal{E}_{\text {Lker }(\pi)}(x)=\mathcal{E}_{\mathcal{P}(\mathbf{h})}(x)-\mathcal{E}_{\mathcal{P}(\mathbf{k})}(x) .
$$

Since $\operatorname{Lker}(\pi)$ is a Lie submonoid of $\mathcal{P}(\mathbf{h})$, the submonoid of $\mathbf{h}$ generated by $\operatorname{Lker}(\pi)$ identifies with $\mathcal{U}(\operatorname{Lker}(\pi))$. Therefore, as above, the generating series for the latter submonoid is

$$
\exp \left(\mathcal{E}_{\mathrm{Lker}(\pi)}(x)\right)=\exp \left(\mathcal{E}_{\mathcal{P}(\mathbf{h})}(x)-\mathcal{E}_{\mathcal{P}(\mathbf{k})}(x)\right)=\mathcal{E}_{\mathrm{Hker}(\pi)}(x),
$$

which is the desired equality. 
Remark. The results of this section hold also for connected (not necessarily graded or finite dimensional) Hopf algebras. See [5, Example 4.20] for a proof of Proposition 32 in this setting. The proof above used finite dimensionality of the (components of the) species, but this hypothesis is not necessary. The proof in [5] may be adapted to yield the result for arbitrary species.

5.2. The Lie kernel of $\pi: \mathbf{L} \rightarrow \mathbf{E}$. We return to the discussion in Example 23. The primitive elements of the Hopf monoids $\mathbf{E}$ and $\mathbf{L}$ are described in [2, Example 11.44]. We have that

$$
\mathcal{P}(\mathbf{E})=\mathbf{X} \quad \text { and } \quad \mathcal{P}(\mathbf{L})=\mathbf{L i e}
$$

where $\mathbf{X}$ is the species of singletons,

$$
\mathbf{X}[I]= \begin{cases}\mathbb{k} & \text { if }|I|=1 \\ 0 & \text { otherwise }\end{cases}
$$

and Lie is the species underlying the Lie operad. It follows that the Lie kernel of the canonical morphism $\pi: \mathbf{L} \rightarrow \mathbf{E}$ is given by

$$
\operatorname{Lker}(\pi)[I]= \begin{cases}\operatorname{Lie}[I] & \text { if }|I| \geq 2 \\ 0 & \text { otherwise. }\end{cases}
$$

Before moving on to the Hopf kernel of $\pi$, we provide some more information on the species Lie.

Let $I$ be a finite nonempty set and $n=|I|$. It is known that the space $\mathbf{L i e}[I]$ is of dimension $(n-1)$ !. We proceed to describe a linear basis indexed by cyclic orders on $I$. A cyclic order on $I$ is an equivalence class of linear orders on $I$ modulo the action

$$
i_{1}|\cdots| i_{n-1}\left|i_{n} \mapsto i_{n}\right| i_{1}|\cdots| i_{n-1}
$$

of the cyclic group of order $n$. Each class has $n$ elements so there are $(n-1)$ ! cyclic orders on $I$. We use $(b, a, c)$ to denote the equivalence class of the linear order $b|a| c$.

We fix a finite nonempty set $I$ and choose a linear order $\ell_{0}$ on $I$, say

$$
\ell_{0}=i_{1}\left|i_{2}\right| \cdots \mid i_{n}
$$

The basis of $\mathbf{L i e}[I]$ will depend on this choice. Given a cyclic order $\gamma$ on $I$, let $S$ be the subset of $I$ consisting of the elements encountered when traversing the cycle from $i_{1}$ to $i_{2}$ clockwise, including $i_{1}$ but excluding $i_{2}$ (these are the first and second elements in $\ell_{0}$, respectively). Let $T$ consist of the remaining elements (from $i_{2}$ to $i_{1}$ ). Note that $i_{1} \in S$ and $i_{2} \in T$, so both $S$ and $T$ are nonempty. The cyclic order $\gamma$ on $I$ induces cyclic orders on $S$ and $T$. We denote them by $\left.\gamma\right|_{S}$ and $\left.\gamma\right|_{T}$. An element $p_{\gamma} \in \mathbf{L}[I]$ is defined recursively by

$$
p_{\gamma}:=\left[p_{\left.\gamma\right|_{S}}, p_{\left.\gamma\right|_{T}}\right]=p_{\left.\gamma\right|_{S}} \cdot p_{\left.\gamma\right|_{T}}-p_{\left.\gamma\right|_{T}} \cdot p_{\left.\gamma\right|_{S}} .
$$

The elements $p_{\left.\gamma\right|_{S}} \in \mathbf{L}[S]$ and $p_{\left.\gamma\right|_{T}} \in \mathbf{L}[T]$ are themselves defined with respect to the induced linear orders $\left.\left(\ell_{0}\right)\right|_{S}$ and $\left.\left(\ell_{0}\right)\right|_{T}$. The recursion starts with the case when $I$ is a singleton $\{a\}$. In this case, we set

$$
p_{(a)}:=a \in \mathbf{L}[a]
$$

(the unique linear order). 
Clearly $a \in \mathbf{L}[a]$ is a primitive element. Since the primitive elements are closed under commutators, we have $p_{\gamma} \in \mathbf{L i e}[I]$. Moreover, we have the following.

Proposition 33. For fixed $I$ and $\ell_{0}$ as above, the set

$$
\left\{p_{\gamma} \mid \gamma \text { is a cyclic order on } I\right\}
$$

is a linear basis of $\mathbf{L i e}[I]$.

Proof. The construction of the elements $p_{\gamma}$ is a reformulation of the familiar construction of the Lyndon basis of a free Lie algebra [13, 24, 25]. Reading the elements of the cyclic order $\gamma$ clockwise starting at the minimum of $\ell_{0}$ gives rise to a Lyndon word on $I$ (without repeated letters). The cyclic orders $\left.\gamma\right|_{S}$ and $\left.\gamma\right|_{T}$ give rise to the Lyndon words in the canonical factorization of this Lyndon word.

For example, suppose that $I=\{a, b, c, d\}, \ell_{0}=a|b| c \mid d$ and $\gamma=(b, a, c, d)$. Then

$$
\begin{aligned}
p_{(b, a, c, d)} & =\left[p_{(a, c, d)}, p_{(b)}\right]=\left[\left[p_{(a)}, p_{(c, d)}\right], p_{(b)}\right]=\left[\left[p_{(a)},\left[p_{(c)}, p_{(d)}\right]\right], p_{(b)}\right]=[[a,[c, d]], b] \\
& =a|c| d|b-a| d|c| b-c|d| a|b+d| c|a| b-b|a| c|d+b| a|d| c+b|c| d|a-b| d|c| a .
\end{aligned}
$$

Remark. The vector species Lie is not the linearization of the set species of cycles. Note also that, for a general bijection $\sigma: I \rightarrow J$, the $p$-basis of Lie $[I]$ will not map to the $p$-basis of $\mathbf{L i e}[J]$ under $\mathbf{L}[\sigma]$.

5.3. The Hopf kernel of $\pi: \mathbf{L} \rightarrow \mathbf{E}$. The above description (5) of the Lie kernel of $\pi: \mathbf{L} \rightarrow \mathbf{E}$ together with Proposition 32 imply that the Hopf kernel of $\pi$ is given by

$$
\operatorname{Hker}(\pi)[I]=\sum_{k \geq 1} \sum_{\substack{S_{1} \sqcup \cdots \sqcup S_{k}=I \\\left|S_{r}\right| \geq 2 \forall r}} \operatorname{Lie}\left[S_{1}\right] \cdots \operatorname{Lie}\left[S_{k}\right] .
$$

An element in $\mathbf{L i e}\left[S_{1}\right] \cdots \mathbf{L i e}\left[S_{k}\right]$ is a $k$-fold product of primitive elements $x_{r} \in \mathbf{L i e}\left[S_{r}\right]$; each $S_{r}$ must have at least 2 elements. We proceed to describe a linear basis for $\operatorname{Hker}(\pi)[I]$.

As in Section 5.2, we fix a linear order $\ell_{0}=i_{1}\left|i_{2}\right| \cdots \mid i_{n}$ on $I$. The basis will be indexed by derangements of $\ell_{0}$. A derangement of $\ell_{0}$ is a linear order $\ell=j_{1}\left|j_{2}\right| \cdots \mid j_{n}$ on $I$ such that $i_{r} \neq j_{r}$ for all $r=1, \ldots, n$.

View linear orders as bijections $[n] \rightarrow I$ and define $\sigma:=\ell \circ \ell_{0}^{-1}$. Then $\sigma$ is a permutation of $I$ and $\ell$ is a derangement of $\ell_{0}$ precisely when $\sigma$ has no fixed points.

Let $\ell$ be a derangement of $\ell_{0}$ and $\sigma$ the associated permutation. Let $S_{1}, \ldots, S_{k}$ be the orbits of $\sigma$ on $I$ labeled so that

$$
\min S_{1}<\cdots<\min S_{k} \text { according to } \ell_{0},
$$

and let $\gamma_{r}$ be the cyclic order on $S_{r}$ induced by $\sigma$. In other words, $\sigma=\gamma_{1} \cdots \gamma_{k}$ is the factorization of $\sigma$ into cycles, ordered in this specific manner.

Employing the $p$-basis of Lie from Section 5.2 (defined with respect to $\ell_{0}$ and the orders induced by $\ell_{0}$ on subsets of $I$ ), we define an element $p_{\ell} \in \mathbf{L}[I]$ by

$$
p_{\ell}:=p_{\gamma_{1}} \cdots p_{\gamma_{k}} .
$$

By assumption, $\left|S_{r}\right| \geq 2$ for all $r$. Hence $p_{\gamma_{r}} \in \operatorname{Lker}(\pi)\left[S_{r}\right]$ and $p_{\ell} \in \operatorname{Hker}(\pi)[I]$. 
For example, let $I=\{e, i, m, s, t\}, \ell_{0}=s|m| i|t| e$ and $\ell=i|t| e|m| s$. Then

$$
\sigma=(s, i, e)(m, t), \quad S_{1}=\{i, e, s\}, \quad S_{2}=\{m, t\},
$$

and

$$
p_{\ell}=p_{(s, i, e)} p_{(m, t)}=\left[p_{(s)}, p_{(i, e)}\right] p_{(m, t)}=[s,[i, e]][m, t] .
$$

Proposition 34. For fixed $I$ and $\ell_{0}$ as above, the set

$$
\left\{p_{\ell} \mid \ell \text { is a derangement of } \ell_{0}\right\}
$$

is a linear basis of $\operatorname{Hker}(\pi)[I]$.

Proof. This follows from Proposition 33 and the Poincaré-Birkhoff-Witt theorem.

Example 35. We describe the $p$-basis of $\operatorname{Hker}(\pi)[I]$ in low cardinalities. Throughout, we choose

$$
\ell_{0}=a|b| c \mid \cdots .
$$

The space $\operatorname{Hker}(\pi)[a, b]$ is 1-dimensional, linearly spanned by

$$
p_{b \mid a}=p_{(a, b)}=[a, b] .
$$

The space $\operatorname{Hker}(\pi)[a, b, c]$ is 2-dimensional, linearly spanned by

$$
\begin{aligned}
& p_{b|c| a}=p_{(a, b, c)}=\left[p_{(a)}, p_{(b, c)}\right]=[a,[b, c]], \\
& p_{c|a| b}=p_{(a, c, b)}=\left[p_{(a, c)}, p_{(b)}\right]=[[a, c], b] .
\end{aligned}
$$

The space $\operatorname{Hker}(\pi)[a, b, c, d]$ is 9 -dimensional. There are 6 basis elements corresponding to 4-cycles, such as

$$
p_{c|a| d \mid b}=p_{(a, c, d, b)}=[[a,[c, d]], b],
$$

and 3 basis elements corresponding to products of two 2-cycles, such as

$$
p_{b|a| d \mid c}=p_{(a, b)} p_{(c, d)}=[a, b] \cdot[c, d] .
$$

APPENDIX

The following fact was referred to in the last remark in Section 4.

Proposition 36. Let $H$ be a connected (not necessarily graded) Hopf algebra over a field of characteristic 0 . Then $H$ is a domain.

This result is proven in [34, Lemma 1.8(a)], where it is attributed to Le Bruyn. We provide a different proof here.

Proof. Let $K$ denote the associated graded Hopf algebra with respect to the coradical filtration of $H$. Since $H$ is connected, $K$ is commutative [3, Remark 1.7]. Now by [23, Proposition 1.2.3], $K$ embeds in a shuffle Hopf algebra. The latter is a free commutative algebra [23, Corollary 3.1.2], hence a domain. It follows that $K$ and hence also $H$ are domains.

Over a field of positive characteristic, the restricted universal enveloping algebra $\mathfrak{u}(\mathfrak{g})$ of a finite dimensional nonzero Lie algebra $\mathfrak{g}$ is a connected Hopf algebra that is not a domain. Indeed, in this case $u(\mathfrak{g})$ is finite dimensional [8, Theorem V.12] and so has a nontrivial idempotent, being a Hopf algebra with integrals [18, Theorem 2.1.3]. 


\section{REFERENCES}

[1] Eiichi Abe. Hopf algebras, volume 74 of Cambridge Tracts in Mathematics. Cambridge University Press, Cambridge, 1980. Translated from the Japanese by Hisae Kinoshita and Hiroko Tanaka.

[2] Marcelo Aguiar and Swapneel Mahajan. Monoidal functors, species and Hopf algebras, volume 29 of CRM Monograph Series. American Mathematical Society, Providence, RI, 2010.

[3] Marcelo Aguiar and Frank Sottile. Cocommutative Hopf algebras of permutations and trees. J. Algebraic Combin., 22:451-470, 2005.

[4] François Bergeron, Gilbert Labelle, and Pierre Leroux. Combinatorial species and tree-like structures, volume 67 of Encyclopedia of Mathematics and its Applications. Cambridge University Press, Cambridge, 1998. Translated from the 1994 French original by Margaret Readdy, with a foreword by Gian-Carlo Rota.

[5] Robert J. Blattner, Miriam Cohen, and Susan Montgomery. Crossed products and inner actions of Hopf algebras. Trans. Amer. Math. Soc., 298(2):671-711, 1986.

[6] Jacob C. E. Dekker. Myhill's theory of combinatorial functions. Modern Logic, 1(1):3-21, 1990.

[7] Adriano M. Garsia and Nolan Wallach. Qsym over Sym is free. J. Combin. Theory Ser. A, 104(2):217-263, 2003.

[8] Nathan Jacobson. Lie algebras. Dover Publications Inc., New York, 1979. Republication of the 1962 original.

[9] André Joyal. Une théorie combinatoire des séries formelles. Adv. in Math., 42(1):1-82, 1981.

[10] Irving Kaplansky. Bialgebras. Lecture Notes in Mathematics. Department of Mathematics, University of Chicago, Chicago, Ill., 1975.

[11] Richard G. Larson and Moss E. Sweedler. An associative orthogonal bilinear form for Hopf algebras. Amer. J. Math., 91:75-94, 1969.

[12] Aaron Lauve and Sarah K. Mason. Qsym over sym has a stable basis. J. Combin. Theory Ser. A, 118(5):1661-1673, 2011.

[13] M. Lothaire. Combinatorics on words. Cambridge Mathematical Library. Cambridge University Press, Cambridge, 1997. With a foreword by Roger Lyndon and a preface by Dominique Perrin. Corrected reprint of the 1983 original, with a new preface by Perrin.

[14] Volodymyr Lyubashenko. Modular transformations for tensor categories. J. Pure Appl. Algebra, 98(3):279-327, 1995.

[15] Ian G. Macdonald. Symmetric functions and Hall polynomials. Oxford Mathematical Monographs. The Clarendon Press Oxford University Press, New York, second edition, 1995. With contributions by A. Zelevinsky, Oxford Science Publications.

[16] Akira Masuoka. Freeness of Hopf algebras over coideal subalgebras. Comm. Algebra, 20(5):1353$1373,1992$.

[17] Matías Menni. Algebraic categories whose projectives are explicitly free. Theory Appl. Categ., 22:No. 20, 509-541, 2009.

[18] Susan Montgomery. Hopf algebras and their actions on rings, volume 82 of CBMS Regional Conference Series in Mathematics. Published for the Conference Board of the Mathematical Sciences, Washington, DC, 1993.

[19] John Myhill. Recursive equivalence types and combinatorial functions. Bull. Amer. Math. Soc., 64:373-376, 1958.

[20] Warren D. Nichols and M. Bettina Zoeller. A Hopf algebra freeness theorem. Amer. J. Math., 111(2):381-385, 1989.

[21] Ulrich Oberst and Hans-Jürgen Schneider. Untergruppen formeller Gruppen von endlichem Index. J. Algebra, 31:10-44, 1974.

[22] David E. Radford. Pointed Hopf algebras are free over Hopf subalgebras. J. Algebra, 45(2):266-273, 1977.

[23] David E. Radford. A natural ring basis for the shuffle algebra and an application to group schemes. J. Algebra, 58(2):432-454, 1979. 
[24] Christophe Reutenauer. Free Lie algebras, volume 7 of London Mathematical Society Monographs. New Series. The Clarendon Press Oxford University Press, New York, 1993. Oxford Science Publications.

[25] Christophe Reutenauer. Free Lie algebras. In Handbook of algebra, Vol. 3, pages 887-903. NorthHolland, Amsterdam, 2003.

[26] J. C. Rosales and P. A. García-Sánchez. Numerical semigroups, volume 20 of Developments in Mathematics. Springer, New York, 2009.

[27] Hans-Jürgen Schneider. Principal homogeneous spaces for arbitrary Hopf algebras. Israel J. Math., 72(1-2):167-195, 1990. Hopf algebras.

[28] Hans-Jürgen Schneider. Normal basis and transitivity of crossed products for Hopf algebras. $J$. Algebra, 152(2):289-312, 1992.

[29] Neil J. A. Sloane. The on-line encyclopedia of integer sequences. Published electronically at www.research . att. com/ njas/sequences/, OEIS.

[30] Yorck Sommerhäuser. On Kaplansky's conjectures. In Interactions between ring theory and representations of algebras (Murcia), volume 210 of Lecture Notes in Pure and Appl. Math., pages 393-412. Dekker, New York, 2000.

[31] Richard P. Stanley. Enumerative combinatorics. Vol. 2, volume 62 of Cambridge Studies in Advanced Mathematics. Cambridge University Press, Cambridge, 1999. With a foreword by GianCarlo Rota and Appendix 1 by Sergey Fomin.

[32] Mitsuhiro Takeuchi. Relative Hopf modules - equivalences and freeness criteria. J. Algebra, 60(2):452-471, 1979.

[33] Mitsuhiro Takeuchi. Finite Hopf algebras in braided tensor categories. J. Pure Appl. Algebra, 138(1):59-82, 1999.

[34] D. G. Wang, J. J. Zhang, and G. Zhuang. Hopf algebras of GK-dimension two with vanishing Ext-group. ArXiv e-prints, April 2011.

(Aguiar) Department of Mathematics, Texas A\&M University, College Station, TX 77843

E-mail address: maguiar@math.tamu.edu

URL: http://www.math.tamu.edu/ maguiar

(Lauve) Department of Mathematics and Statistics, Loyola University Chicago, Chicago, IL 60660

E-mail address: lauve@math.luc.edu

$U R L$ : http://www. math.luc. edu/ lauve 